\title{
Screening and identification of the nucleic acid aptamers in nasopharyngeal carcinoma
}

\author{
W.-X. Chen ${ }^{1,2}$, K.-H. Zhang ${ }^{2}$, X.-S. Zou ${ }^{1}$, Y.-Q. Chen ${ }^{1}$ and J.-G. Li ${ }^{1}$ \\ ${ }^{1}$ First Affiliated Hospital of Nanchang University, Nanchang, China \\ ${ }^{2}$ Tumor Hospital of Jiangxi Province, Nanchang, China \\ Corresponding authors: K.-H. Zhang / J.-G. Li \\ E-mail: yfyzkh@sina.com / lijingao@hotmail.com
}

Genet. Mol. Res. 12 (4): 6850-6857 (2013)

Received November 21, 2012

Accepted May 14, 2013

Published December 19, 2013

DOI http://dx.doi.org/10.4238/2013.December.19.3

\begin{abstract}
To screen the nucleic acid aptamers of the EB virus-positive nasopharyngeal carcinoma cells, we used SELEX technology and synthesized in vitro a 78-nucleotide random DNA library. We used normal nasopharyngeal epithelial cells and EB virus-positive low differentiated nasopharyngeal carcinoma cells as target to conduct 10 cycles of screening, cloning, sequencing, and identification of the aptamers. The fluorescence produced by the combination of the sub-library and the target cells gained intensity gradually with the increase in the number of screening cycles, indicating elevated binding capacity. The cluster analysis showed that the aptamers can be divided into three families, with two of the families having the common conserved sequence. In this study, by screening nucleic acid aptamers for affinity and specificity, we established an initial aptamer library for EB virus-positive nasopharyngeal carcinoma cells.
\end{abstract}

Key words: SELEX; Nasopharyngeal carcinoma; Aptamer 


\section{INTRODUCTION}

SELEX (systematic evolution of ligands by exponential enrichment), is a new combinatorial chemistry technique for producing oligonucleotides of either single-stranded DNA or RNA that specifically bind to target ligands (Tuerk and Gold, 1990; Ellington and Szostak, 1990). The process begins with the synthesis of a very large oligonucleotide library consisting of randomly generated sequences of fixed length flanked by constant 5 '- and 3 '-ends that serve as primers. The technique has been used to evolve nucleic acid aptamers of extremely high binding affinity to a variety of target ligands, such as small molecules, proteins, nucleic acids, and even cells, tissues and organisms. Clinical uses of the technique are suggested by aptamers that bind tumor markers.

SELEX has proven to be an excellent tool for finding nucleotide molecules that have a high affinity for a particular target from a random pool under specific conditions. The subtractive SELEX techonology is an anti-screening experiment to identify the difference between two target molecule nucleic acid aptamers and to obtain the filters through the same oligonucleotide library. The cell SELEX technology is one kind of composite target SELEX technology whose screening target is not a single pure protein or small molecule but the mixture of a variety of target molecules. In this study, we screened the Epstein-Barr (EB) virus-positive nasopharyngeal carcinoma cells with the subtractive SELEX technology and the cell SELEX technology.

\section{MATERIAL AND METHODS}

\section{Library and primers}

The DNA screening library was randomly synthesized by SELEX (Shanghai Biological Engineering Technology Co. Ltd, Shanghai, China). Both ends of the library were fixed primers with the random sequences of 40 nucleotides in the middle (5'-ACC GAC CGT GCT GGA CTC T (-40 nt-) AGT ATG AGC GAG CGT TGC G-3'). PCR primers were designed with the Primer Premier 5.0 software. The upstream primer 5' was labeled with the FITC fluorescence. The sequences of the upstream (B1) and downstream (B2) primers were: 5'-FITC-ACC GAC CGT GCT GGA CTC T-3' and 5'-CGC AAC GCT CGC TCA TAC T-3', respectively.

\section{Cell lines and reagents}

The normal nasopharyngeal epithelial cell lines (NP69) and the EB virus-positive low differentiated nasopharyngeal carcinoma cell lines (C666-1) were kindly provided by Professor Zeng Mu-Sheng of the Affiliated Cancer Prevention Center of the Zhong Shan University. Keratinocyte-SFM and RPMI 1640 medium were purchased from GIBCO; fetal bovine serum obtained from Hangzhou Evergreen; Dulbecco's phosphate buffer and yeast tRNA from Sigma.

\section{SELEX screening procedure}

The SELEX screening procedure was performed according to Fang and Tan (2010). 1) Pretreatment of the library: the single-stranded DNA (1.25 nmol) was dissolved in $500 \mu \mathrm{L}$ 
binding buffer. Heat denatured at $95^{\circ} \mathrm{C}$ for $5 \mathrm{~min}$, and subsequently cooled with an ice bath for 10 min. 2) Pretreatment of the cells (NP69 and C666-1): cells were washed 3 times with the washing buffer (including $8 \mathrm{~g} / \mathrm{L} \mathrm{NaCl}, 0.2 \mathrm{~g} / \mathrm{L} \mathrm{KCl}, 0.14 \mathrm{~g} / \mathrm{L} \mathrm{CaCl}, 0.1 \mathrm{~g} / \mathrm{L} \mathrm{MgCl}_{2}, 1.42 \mathrm{~g} / \mathrm{L}$ $\mathrm{Na}_{2} \mathrm{HPO}_{4}, 0.2 \mathrm{~g} / \mathrm{L} \mathrm{KH}_{2} \mathrm{PO}_{4}, 0.35 \mathrm{~g} / \mathrm{L} \mathrm{NaHCO}_{3}, 4.5 \mathrm{~g} / \mathrm{L}$ glucose, and $0.475 \mathrm{~g} / \mathrm{L} \mathrm{MgCl}_{2}$ ), then digested with $0.53 \mathrm{mM}$ EDTA solution and collected. 3) Subtractive screening: the pretreated library and the NP69 control cells $\left(2 \times 10^{6}\right)$ were incubated at $4{ }^{\circ} \mathrm{C}$ for $60 \mathrm{~min}$. The nucleic acids, which did not bind the NP69 cells, were collected and 4) used as a template to perform an asymmetric PCR amplification with the FITC-labeled primers and recover the single chain as a sub-library for a second round of screening. 5) Screening: this sub-library was dissolved in binding buffer, then processed as follows: $95^{\circ} \mathrm{C}$ heat denaturation for $5 \mathrm{~min}$, ice bath for 10 min, incubation with $\mathrm{C} 666$ cells at $4^{\circ} \mathrm{C}$ for $30 \mathrm{~min}$, washing buffer, $95^{\circ} \mathrm{C}$ heat denaturation for 5 min. 6) The meticulousness of the screening conditions was increased to obtain a highaffine, highly specific aptamer library. Screening time was reduced from 60 to $30 \mathrm{~min}$, the volume of the washing liquid was increased from 0.5 to $1.5 \mathrm{~mL}$, the washing times gradually from 1 to 5, and the proportion of nucleic acid and cells from 100 to 400. FBS (20\%) and 50 to 300 times yeast tRNA were added to reduce non-specific binding of the library. The screening process was terminated after 10 cycles until the sub-library and the target cell binding strength no longer increased.

\section{Asymmetric PCR method}

Conditions were as follows for 20 cycles: $94^{\circ} \mathrm{C}$ pre-denaturation for $3 \mathrm{~min}, 94^{\circ} \mathrm{C}$ for $45 \mathrm{~s}, 68^{\circ} \mathrm{C}$ for $90 \mathrm{~s}$. ssDNA was extracted from a denaturing gel and used as a sub-library for the next round of screening. Dilutions of sub-library series were made and sub-data amount was detected by fluorescent quantitative PCR.

\section{Analysis of the sub-library with the flow cytometry}

The method for flow cytometry was perfomed according to the literatur described previously (Gopinath, 2007).

\section{Cloning, sequencing and analysis of the secondary structure}

The ssDNA was obtained after 10 screening, the dsDNA amplified by PCR, and a single band at $78 \mathrm{bp}$ targeted. Samples were delivered to the Shanghai Sangon for cloning and sequencing. The RNAstructure software was used for analyzing the secondary structure of the sequence, the Clustal X software was used to compare the sequence homology.

\section{Affinity test for the aptamers}

C666 $\left(3 \times 10^{5}\right)$ cells were resuspended in $400 \mu \mathrm{L}$ binding buffer and were added 100 $\mu \mathrm{L}$ aptamers or library with 6 concentration gradients labeled by the FITC. The cells were kept in the ice bath protected from the light for $50 \mathrm{~min}$, washed twice with $0.7 \mathrm{~mL}$ binding buffer (the washing buffer metioned above plus $1 \mathrm{~g} / \mathrm{L}$ BSA and $0.1 \mathrm{~g} / \mathrm{L}$ yeast tRNA) and resuspended in $0.4 \mathrm{~mL}$ binding buffer. Cytometry was performed within $30 \mathrm{~min}$. All the affinity detections 
were repeated once and the average fluorescence intensity, for each concentration of aptamer, was taken to calculate the aptamers affinity. The dissociation constant of the aptamers and the target cells binding were obtained using the Graphpad prism software.

\section{RESULTS}

\section{Asymmetric PCR method for harvesting the single-stranded DNA library}

The two-step method of asymmetric PCR amplification can provide a good yield of ssDNA. The denaturing gel can effectively recycle the ssDNA for the next round of screening. Results are shown in Figure 1.

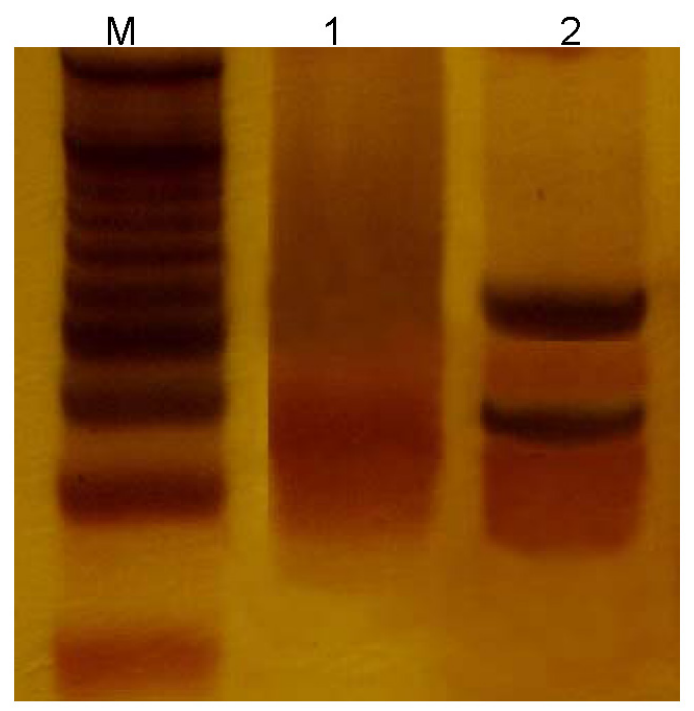

Figure 1. Asymmetric PCR amplification to acquire the sub-library. Lane $M=20$-bp marker; lane $1=$ recycled sub-database; lane 2 = PCR product.

\section{Monitoring of the aptamer library-enrichment process}

Among all the SELEX screening methods targeting cells, flow cytometry having the advantages of faster speed, larger cell number, and quantitative accuracy has become the best tool for monitoring the library enrichment. We successfully monitored the enrichment of the aptamer library by flow cytometry (Figure 2).

\section{Nucleic acid aptamer cloning, sequencing and structural analysis}

For PCR amplification of the ssDNA aptamer library after the 10 stages of screening, we used non-FITC-labeled primers. The resulting dsDNA showed a single band at the predicted size. Samples were then sent to the biotech companies for cloning and sequencing. We used the RNAstructure software to simulate the secondary structure of the aptamer, which resulted 
to have a wide variety of secondary structure. The simulated secondary structure is shown in Figure 3A. We used the Clustal X to make the aptamer sequence comparison. The first structural analysis showed no common conserved sequence, but sequences can be divided into three families: two having the homologous sequence, and one family not (Figure 3B and C).

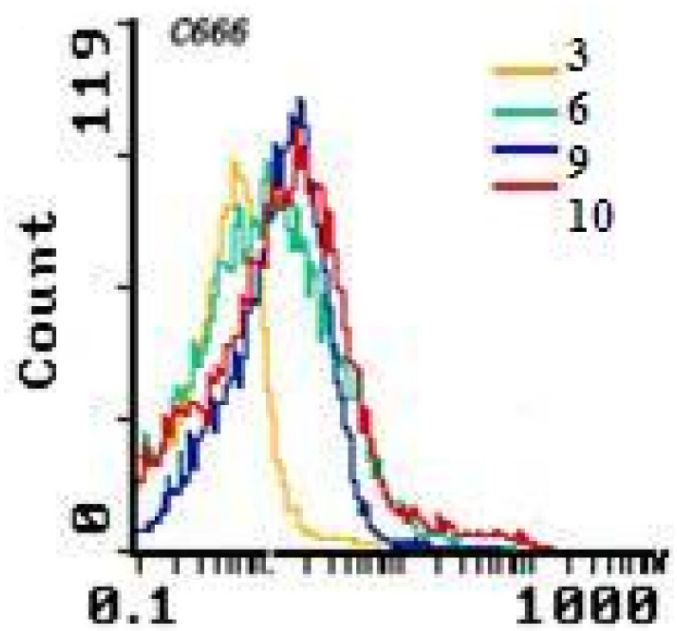

Figure 2. Aptamer library enrichment showing the combined flow fluorescence intensity detection of the nasopharyngeal carcinoma cell line C666 with the library in each stage.

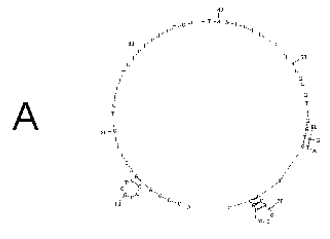

2

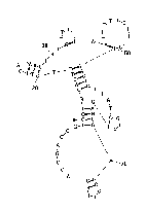

17

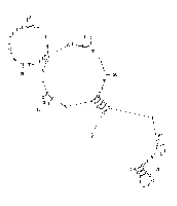

18
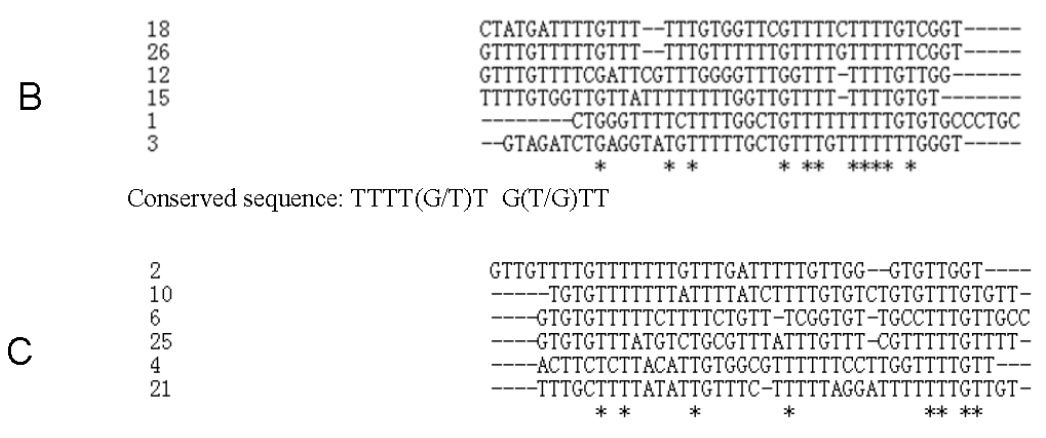

Conserved sequence: TT(T/G)GT

Figure 3. Part of the simulated secondary structure of the aptamer and the homology results of the aptamer. A. Secondary structure of the aptamer. B. Homology results of the aptamer in family 1 . C. Homology results of the aptamer in family 2 . 


\section{Affinity test of the aptamer}

The affinity of two aptamers was tested in each family. All aptamers showed a certain affinity to the C666 cells. The Kd values of the aptamers were tested with the Graphpad prism suitable aptamer saturation curve (Figure 4). The Kd values of the aptamers 2, 6, 8, 12, 3, 5 combined with the nasopharyngeal carcinoma cell C666 were 151.5, 401.9, 418.5, 171.1, 148.4 , and $488.2 \mathrm{nM}$. The Kd value is inversely associated with the aptamer affinity, meaning that the lower the $\mathrm{Kd}$ values, the greater the aptamer affinity. Therefore, the aptamer $2(\mathrm{Kd}=$ $151.5 \mathrm{nM}$ ) showed the highest affinity with the C666 cells.

\section{C666 cell}

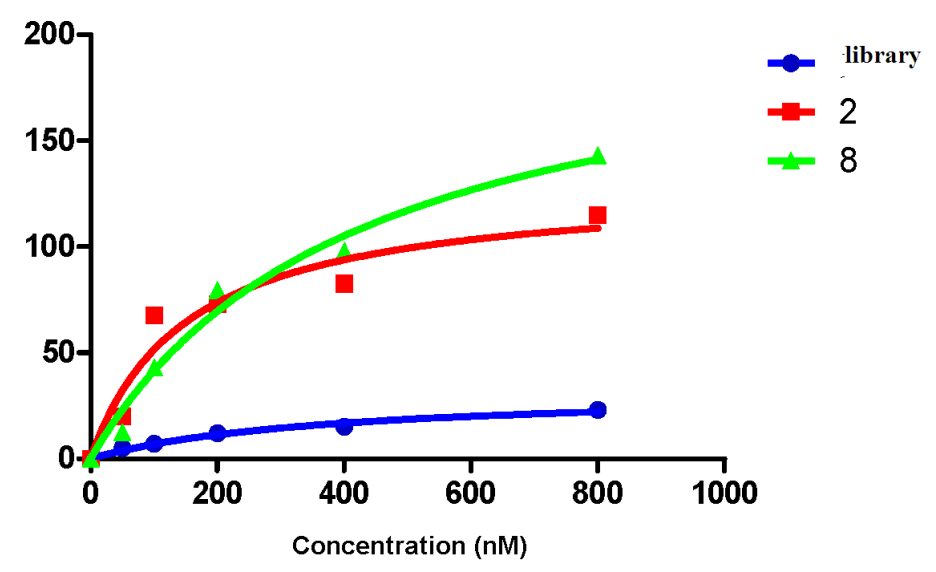

Figure 4. Aptamer saturation curve.

\section{DISCUSSION}

The cell SELEX is a derivative of the SELEX technology, with the prominent advantage of finding out the aptamers, which can specifically recognize the unknown molecules on the cell surface (Hess et al., 2000; Faria and Ulrich, 2002). Although aptamers share many functional similarities with antibodies, the former possess several key advantages. Aptamers are chemically synthesized in vitro, therefore easier and more economical to produce, convenient in storage and transportation, can be chemically altered depending on research necessity, show no toxicity and immunogenicity, and have increased half-life in biological fluids and improved tissue penetration (Ulrich et al., 2004). Aptamers have a prospective wide range of applications in the tumor diagnosis and treatment (Gold, 1995; Cerchia et al., 2009). Shangguan et al (2006) found the specific aptamer of the mouse hepatoma cells using the SELEX technology. Chen et al (2008) screened the nucleic acid aptamer in the small cell lung cancer (SCLC), and strikingly demonstrated the aptamer's specificity in recognition of SCLC cells only, and no non-SCLC cells. Moreover, aptamers generated for certain SCLC cell line can recognize other SCLC cell lines of the same type, but no other subtypes of lung cancer or other types of cancer. 
The NP69 cells are the normal human nasopharyngeal epithelial immortalized cell line, C666 cells derive from patients with nasopharyngeal carcinoma. Despite showing the same morphology, NP69 and C666 cells have different biology, being NP69 normal cells and C666, tumor cells. Therefore, we used the C666 cells as target cells for the screening, and the NP69 cells as a negative control to perform the subtractive screening and obtain the aptamer library of nasopharyngeal carcinoma cells with specific surface markers. Aptamers for the diagnosis and treatment of the nasopharyngeal carcinoma can be further obtained from the aptamer library.

In this study, we used a random DNA library containing 40 bases consisting of $10^{15-16}$ different sequence motifs (Fitzwater and Polisky, 1996; Jayasena, 1999), and using the cell SELEX technology, we found the aptamers with high affinity for the C666 cells. Given the presence of complicated protein binding sites, combination of hydrogen bonds and van der Waals forces, on the surface of the nasopharyngeal carcinoma cells the aptamers can show different affinity. Therefore, in the initial screening under not rigorous conditions, various aptamers had different affinity. Increasing the number of the screening cycles, conditions were stricter, and the sites with a high affinity could be filtered out by the competitive binding of the aptamers. The fluorescence produced by the combination of the sub-library and the C666 cells gained intensity gradually with the increase in the number of filter cycles (Figure 2). After the 6th cycle, the fluorescence intensity produced by the library and the C666 cell binding no longer increased, meaning that the combination of the sub-library and the C666 cells had reached the saturation point.

In this study, after 10 screenings, the random DNA library was significantly enriched. The sequence analyses showed no common conserved sequence in the random primary structure of all aptamers. However, aptamers can be divided into three families, with two of the families having the common conserved sequence. The first family motif was TTTT (G/T) TG $(\mathrm{T} / \mathrm{G}) \mathrm{TT}$, and the second family motif was TT $(\mathrm{T} / \mathrm{G}) \mathrm{GT}$. The motifs played a crucial role in the formation of the aptamer domain functional structure, as each motif represented a different structural domain of the DNA aptamer. Meanwhile, the different combining target sites could be determined.

Cluster analysis of the sequenced aptamers revealed that some aptamers could not cluster, illustrating the diversity of the target molecule and the aptamer binding. The affinity was tested in two aptamers from each family, and the results showed that all the aptamers had certain of affinity to C666 cells. Further analyses will be performed, improving conditions to find out more high-affinity aptamers, and to lay the foundation for the future of the nasopharyngeal cancer diagnosis.

\section{REFERENCES}

Cerchia L, Giangrande PH, McNamara JO and de Francisco V (2009). Cell-specific aptamers for targeted therapies. Methods Mol. Biol. 535: 59-78.

Chen HW, Medley CD, Sefah K, Shangguan D, et al. (2008). Molecular recognition of small-cell lung cancer cells using aptamers. Chem. Med. Chem. 3: 991-1001.

Ellington AD and Szostak JW (1990). In vitro selection of RNA molecules that bind specific ligands. Nature 346: 818-822.

Fang X and Tan W (2010). Aptamers generated from cell-SELEX for molecular medicine: a chemical biology approach. Acc. Chem. Res. 43: 48-57.

Faria M and Ulrich H (2002). The use of synthetic oligonucleotides as protein inhibitors and anticode drugs in cancer therapy: accomplishments and limitations. Curr. Cancer Drug Targets 2: 355-368. 
Fitzwater T and Polisky B (1996). A SELEX primer. Methods Enzymol. 267: 275-301.

Gold L (1995). Oligonucleotides as research, diagnostic, and therapeutic agents. J. Biol. Chem. 270: 13581-13584.

Gopinath SC (2007). Methods developed for SELEX. Anal. Bioanal. Chem. 387: 171-182.

Hess GP, Ulrich H, Breitinger HG, Niu L, et al. (2000). Mechanism-based discovery of ligands that counteract inhibition of the nicotinic acetylcholine receptor by cocaine and MK-801. Proc. Natl. Acad. Sci. U. S. A. 97: 13895-13900.

Jayasena SD (1999). Aptamers: an emerging class of molecules that rival antibodies in diagnostics. Clin. Chem. 45: 1628-1650.

Shangguan D, Li Y, Tang Z, Cao ZC, et al. (2006). Aptamers evolved from live cells as effective molecular probes for cancer study. Proc. Natl. Acad. Sci. U. S. A. 103: 11838-11843.

Tuerk C and Gold L (1990). Systematic evolution of ligands by exponential enrichment: RNA ligands to bacteriophage T4 DNA polymerase. Science 249: 505-510.

Ulrich H, Martins AH and Pesquero JB (2004). RNA and DNA aptamers in cytomics analysis. Cytometry A 59: 220-231. 